\title{
Vascular epiphytes on Pseudobombax (Malvaceae) in rocky outcrops (inselbergs) in Brazilian Atlantic Rainforest: basis for conservation of a threatened ecosystem
}

\author{
Dayvid Rodrigues Couto ${ }^{1,4}$, Henrique Machado Dias ${ }^{1}$, Mirian Cristina Alvarez Pereira ${ }^{2}$, \\ Cláudio Nicoletti de Fraga ${ }^{3} \&$ José Eduardo Macedo Pezzopane ${ }^{1}$
}

\begin{abstract}
This study evaluated the richness of vascular epiphytes on Pseudobombax sp. nov. in three inselbergs in the Atlantic Forest Domain in state of Espírito Santo and evaluated the floristic similarity between the areas. We sampled 111 phorophytes in three regions in the southern of Espírito Santo state and identified 151 species, 77 genera and 21 families of vascular epiphytes, of which the families Orchidaceae and Bromeliaceae showed the highest richness. Non-parametric estimators (Chao 2, Jackknife 1) indicate that 90 and $95 \%$ of species richness of epiphytes was recorded. The most diversified ecological category was the characteristic holoepiphytes. The ratio of the number of epiphytes and number of phorophytes sampled in a montane inselberg, in this study, was greater than the richness of vascular epiphytes found in the rocky outcrops of quartzite, and, in general, different types of Atlantic Domain forests, but smaller in richness for some Dense Ombrophilous Forests of southern Brazil. The three inselberg areas had distinct floras. The high richness, the endemism found, and the number of endangered species of epiphytes demonstrate the important role of Pseudobombax sp. nov., because of its architecture and size, in the maintenance of biodiversity on the inselbergs in southeastern Brazil.
\end{abstract}

Key words: conservation, non-parametric estimators richness, specific phorophyte, vascular flora, industrial granite quarries.

\begin{abstract}
Resumo
Este estudo avaliou a riqueza de epífitas vasculares sobre Pseudobombax sp. nov. em três inselbergs do Domínio Atlântico no estado do Espírito Santo e avaliou a similaridade florística entre as áreas. Foram amostrados 111 forófitos, em três regiões no sul do estado do Espírito Santo e identificados 151 espécies, 77 gêneros e 21 famílias de epífitos vasculares, das quais as famílias Orchidaceae e Bromeliaceae apresentaram maior riqueza. Estimadores não-paramétricos (Chao 2, Jackknife 1) indicam que 90 e $95 \%$ da riqueza específica de epífitas foi registrada. A categoria ecológica mais diversificada foi a dos holoepífitos característicos. A razão entre o número de epífitas e número de forófitos amostrados em um inselberg montano, neste estudo, foi maior do que a riqueza de epífitos encontrados nos campos rupestres, e, em geral, em diferentes tipos de florestas do Domínio Atlântico, mas menor em riqueza para algumas Florestas Ombrófilas Densas do sul do Brasil. As três áreas de inselbergs tinham floras distintas. A elevada riqueza, o endemismo encontrado e o número de espécies ameaçadas dos epífitos evidenciam a importante função de Pseudobombax sp. nov., por sua arquitetura e tamanho, para a manutenção da biodiversidade em inselbergs do sudeste brasileiro.

Palavras-chave: conservação, estimadores não-paramétricos de riqueza, forófito específico, flora vascular, pedreira de granito industrial.
\end{abstract}

\footnotetext{
Universidade Federal do Espírito Santo (CCA/UFES), Programa de Pós-graduação em Ciências Florestais, Depto. Ciências Florestais e da Madeira, Centro de Ciências Agrárias, Av. Governador Lindemberg 316, 29550-000, Jerônimo Monteiro, ES, Brazil.

${ }^{2}$ Universidade Federal do Espírito Santo (CCA/UFES), Depto. Ciências Biológicas, Centro de Ciências Agrárias, Alto Guararema s/n, 29500-000, Alegre, ES, Brazil.

${ }^{3}$ Instituto de Pesquisas Jardim Botânico do Rio de Janeiro, R. Pacheco Leão 915, 22460-030, Rio de Janeiro, RJ, Brazil.

${ }^{4}$ Author for correspondence: dayvidcouto@hotmail.com
} 


\section{Introduction}

Epiphytes represent a strategy since the appearance of trees in the late Devonian and Carboniferous (Dilcher et al. 2004) evolving convergently in a large number of lineages of vascular and non-vascular plants (Gentry \& Dodson 1987). Vascular epiphytes represnt about $9 \%$ of the world's vascular plant flora and are most abundant in tropical rain forests, where they comprise a significant part of the diversity and complexity found in these ecosystems (Krömer et al. 2007; Zotz 2013).

In Brazil, studies on this group of plants are concentrated mainly in the south and southeast regions, especially in forest ecosystems associated with the Atlantic Forest Domain. Several investigators have described the high richness of vascular epiphytic species in these regions, associated with multiple phorophytes (Buzatto et al. 2008; Kersten \& Kuniyoshi 2009; Fontoura et al. 2009; Geraldino et al. 2010; Mania \& Monteiro 2010; Blum et al. 2011) or specific phorophytes (Werneck \& Espírito-Santo 2002; Gonçalves \& Waechter 2003; Obermüller et al. 2012) with predominance of members of the families Orchidaceae and Bromeliaceae, representing the most diverse ecological category of true holoepiphytes, spending their entire life cycle on phorophytes.

The few studies focusing on specific phorophytes in Brazil have shown the importance of these plants for maintaining epiphyte biodiversity. In the present study, we selected Pseudobombax sp. nov. (Malvaceae, Bombacoideae) as a specific phorophyte. This large tree commonly occurs in the Atlantic Forest Domain on granite-gneiss rocky outcrops known as inselbergs, and is usually densely covered by epiphytes. Inselbergs are isolated islands covered by different vegetation within the original forest matrix, and harbor a highly distinctive flora with high species richness and endemism (Barthlott \& Porembski 2000).

Among the three major regions recognized worldwide for vegetation of inselbergs, southeastern Brazil stands out (Porembski 2007). In this context, the inselbergs situated in the southern portion of the state of Espírito Santo, are recognized as priority for floristic studies and the creation of protected area, although knowledge of their biodiversity is still incipient (Martinelli 2007). In addition to these gaps, these environments are highly threatened by industrial granit quarrying. This industry was first established in Brazil in Espírito Santo, which is now the largest stone-processing center in Latin America (Chiodi-Filho 2009). Based on the high species richness and endemism, and on these anthropogenic pressures, the inselbergs of southeastern Brazil are recognized as one of the three most important area for inselberg conservation (Porembski 2007).

In view of the importance of epiphytes to species richness in tropical ecosystems, especially in the Atlantic Forest Domain (Kersten 2010), and of the limited knowledge of epiphytes associated with granite-gneiss inselbergs in Brazil, the present study evaluated the richness of vascular epiphytic species associated with Pseudobombax sp. nov. on three inselbergs of the Atlantic Forest. We also categorized these species according to their ecological relationships with the phorophyte, and evaluated the floristic similarity among the three inselbergs, generating an important basis for conservation of these threatened ecosystems.

\section{Material and Methods}

Study area

Three populations of Pseudobombax sp. nov. occurring on granite-gneiss inselbergs in southern Espírito Santo state were sampled (Fig. 1). In each inselberg 37 phorophytes were sampled (111 total).

Upper montane Inselberg - UMI (Fig. 2a-b) - Granitic-gneissic Inselberg of Pedra Roxa (PR) is located in the Caparaó National Park, municipality of Ibitirama (20'23'43's and $\left.41^{\circ} 44^{\prime} 05^{\prime} \mathrm{W}\right)$, with a sampling area of ca. $2 \mathrm{ha}$, approximate elevation of $1,114 \mathrm{~m}$, in a humid valley on the banks of the Pedra Roxa River, within a matrix of Dense Ombrophilous Montane Forest (Veloso et al. 1991). The climate is Köeppen Cwb (highland tropical climate), with a mean annual rainfall of 1,391 $\mathrm{mm}$ and a mean annual temperature of $17^{\circ} \mathrm{C}$. In Caparaó National Park, Pseudobombax sp. nov. forms large groupings; it is the only tree species found on rocky outcrops, and reaches a mean height of $4.8 \mathrm{~m}( \pm 2.2)$ and mean diameter at $1.3 \mathrm{~m}$ above ground level (diameter at breast height, dbh) of $22.4 \mathrm{~cm}( \pm 17.5)$.

Montane Inselberg - MI (Fig. 2c,e) Granitic-gneissic Inselberg of Pedra dos Pontões (PP) is located in the municipality of Mimoso do Sul (20'56'18'S and 41'32'38'W), between 
700 and $730 \mathrm{~m}$ elevation, surrounded by a human-impacted matrix of fragments of Montane Seasonal Semideciduous Forest (Veloso et al. 1991). The climate is Köeppen Cwb, with a mean annual rainfall of $1,375 \mathrm{~mm}$ and mean annual temperature of $21^{\circ} \mathrm{C}$. In Pedra dos Pontões, sparse stands of Pseudobombax sp. nov. are a prominent feature of the rocky outcrop, with some large individuals, in some cases forming small groups, with a mean height of $7.7 \mathrm{~m}( \pm 3.3)$ and mean dbh of $46.4 \mathrm{~cm}( \pm 31.9)$. In this area, the richness of epiphytic Bromeliaceae is great, as described by Couto et al. (2013).

Submontane Inselberg - SMI (Fig. 2d) Granitic-gneissic Inselberg of Pedra Lisa (PL) is located in the district of Burarama, municipality of Cachoeiro de Itapemirim (20 41'55'S and $41^{\circ} 18^{\prime} 28^{\prime \prime} \mathrm{W}$ ), between 180 and $300 \mathrm{~m}$ elevation, within an anthropogenic matrix of fragments of Submontane Seasonal Semideciduous Forest (Veloso et al. 1991). The climate is Köeppen Cwa, with a mean annual rainfall of $1,293 \mathrm{~mm}$ and mean temperature of $24^{\circ} \mathrm{C}$. In this area, Pseudobombax sp. nov. grows sparsely, directly on the rock, and reaches a mean height of $6.2 \mathrm{~m}$ $( \pm 3.9)$ and mean dbh of $35.8 \mathrm{~cm}( \pm 13.8)$.
Phorophyte sampling

Pseudobombax sp. nov. (Malvaceae; Bombacoideae) shows a morphological (leaves, flowers, fruits and seeds) and evolutionary affinity to Pseudobombax petropolitanum A.Robyns, since they belong to the same clade, which has apparently recently evolved (J.G. Carvalho-Sobrinho, unpublished data). Currently the phorophyte taxon used in the present study is an undescribed species of Pseudobambax and being described with name Pseudobombax rupicola Carvalho-Sobrinho \& D.R. Couto (indet.), referring to strictly rupicolous habit. The individuals sampled were caespitose shrubs or trees, with heights of $2.5 \mathrm{~m}$ up to $15.3 \mathrm{~m}$ with patent branches and large surface roots on the rock.

\section{Data collection and analysis}

To develop the list of epiphytic vascular plants, 111 individuals of Pseudobombax sp. nov. were surveyed. When necessary, the trees were climbed using mountain-climbing techniques adapted to the canopy (Perry 1978).

Epiphytes found fertile were collected, following the usual procedures of floristic

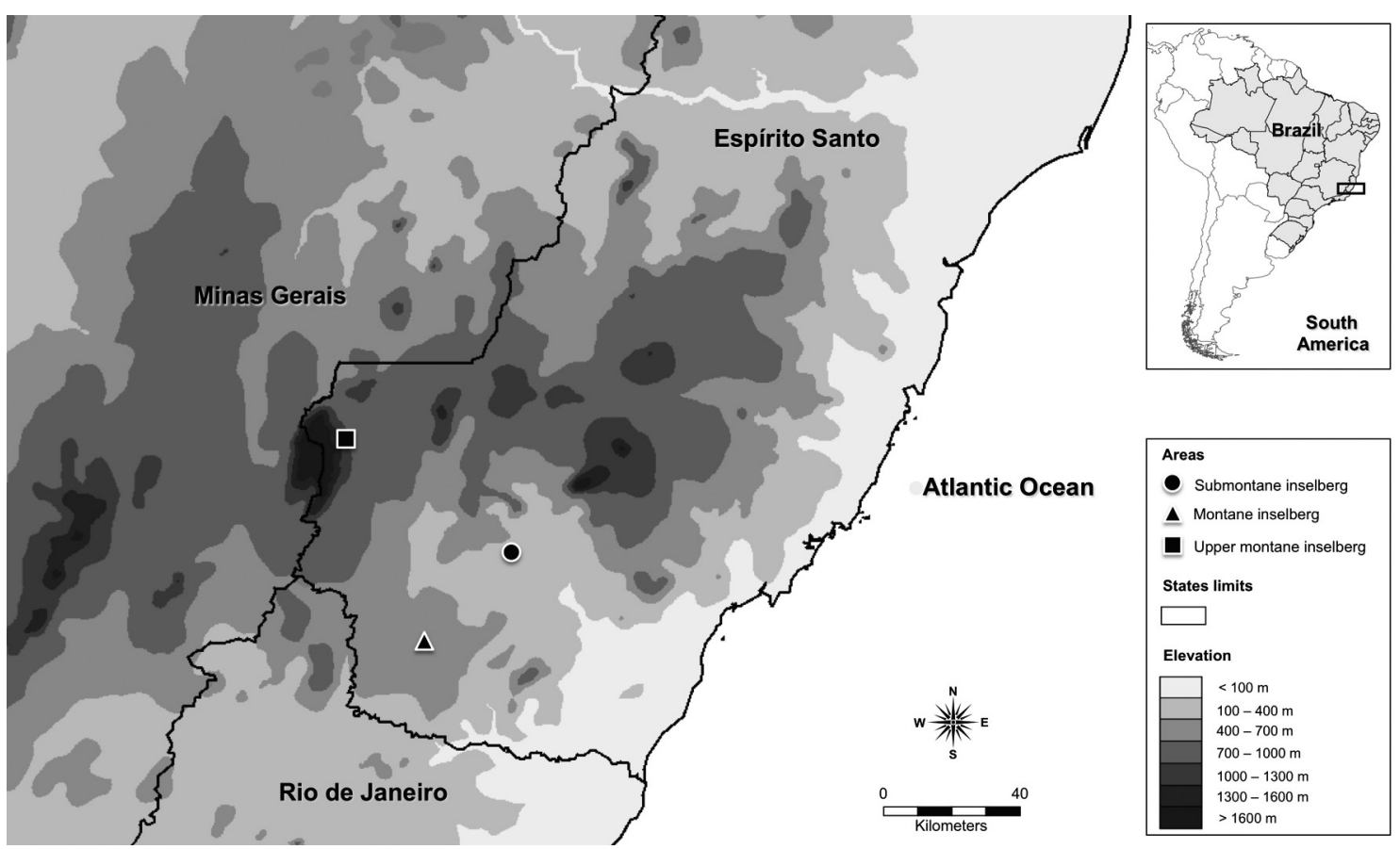

Figure 1 - Location of three populations of Pseudobombax sp. nov. on granitic-gneissic inselbergs in the southern part of Espírito Santo state, where the epiphytic vascular flora was recorded. 

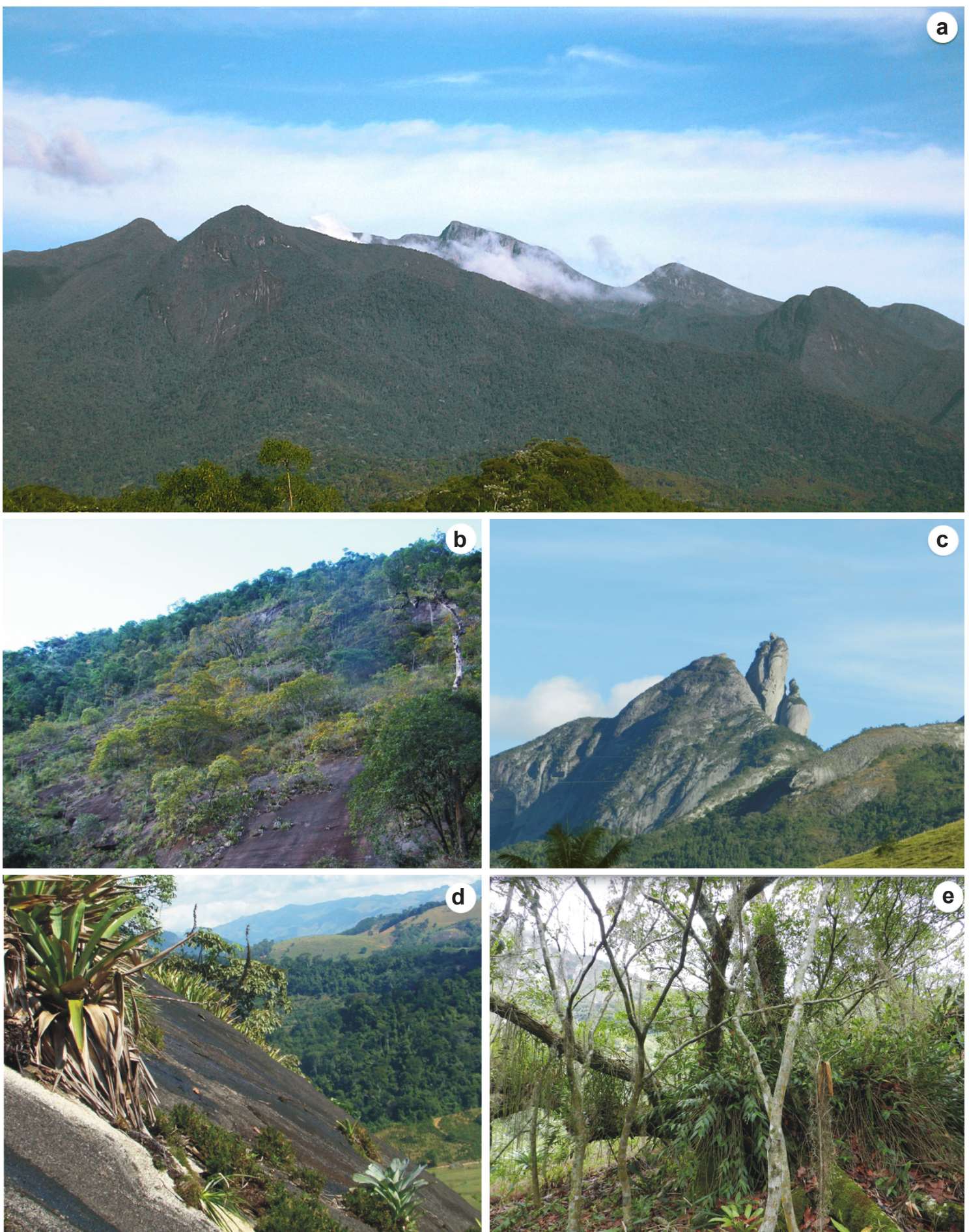

Figure 2 - Occurrence of environments of Pseudobombax sp.nov. in the Brazilian Atlantic Rainforest, where vascular epiphytes were recorded. - a. general view of the vegetation on Caparao National Park; b. general view of population the of Pseudobombax sp.nov. from upper montane inselberg of Pedra Roxa; c. general view of the montane inselberg of Pedra dos Pontões; d. submontane inselberg of Pedra Lisa; e. detail of a phorophyte sampled from Pedra dos Pontões . 
surveys (Mori et al. 1989), with the collections, when possible, made in triplicate, to send to experts on the respective taxa. Specimens were collected sterile when necessary, grown until they flowered, and herborized for later identification.

The botanical material was identified using analytical taxonomic keys, or by comparison with material deposited in herbaria, or by sending duplicate specimens to the specialists. The voucher specimens are deposited mostly in the herbarium of the Museum of Biology Prof. Mello Leitão (MBML) and the material from the most recent surveys (2010-2012) was deposited in the herbarium of the Federal University of Espírito Santo (VIES). Duplicates were sent to the following herbaria: R (National Museum of Rio de Janeiro), RB (Research Institute of the Botanical Garden of Rio de Janeiro), UFRN (Herbarium of the Federal University of Rio Grande do Norte) and VIC (Herbarium of the Federal University of Viçosa).

The circumscription of species in families for angiosperms follows the Angiosperm Phylogeny Group (APG III 2009), Smith et al. (2006) for monilophytes (ferns) and Christenhusz et al. (2011) for lycophytes with taxa names being updated according to supplementary materials in BFG (2015) for angioesperms and Prado et al. (2015) for ferns and lycophytes, and the Missouri Botanical Garden (<http://tropicos.org $>$ ). Abbreviations of author names follow Brummitt \& Powell (1992) and IPNI $(<\mathrm{http}: / /$ www.ipni.org $>)$.

In order to estimate the total number of epiphytic species for each sampled inselberg (SMI, MI and UMI) and for the total sample, rarefaction curves were constructed, based on the presence or absence of epiphytes on phorophytes using three statistical non-parametric estimators: ICE, Chao 2 and Jackknife 1 (Gotelli \& Colwell 2011), performed by 100 random resampling of the data, using EstimateS software (Colwell 2013).

Threatened species are cited according to the Official List of Endangered Species of the Brazilian Flora, through Ministerial Order number 443, of 17 December 2014 (MMA 2014), and specifically for Espírito Santo, as listed by the State Institute of the Environment (Espírito Santo 2005), under the protection of Decree 1499-R, published by Simonelli \& Fraga (2007).
From field observations, the epiphytes were classified into four ecological categories, according to Benzing (1990), with modifications according to Kersten \& Kuniyoshi (2009), as: characteristic holoepiphytes (Epi), facultative holoepiphytes (Fac), accidental holoepiphytes (Aci), hemiepiphytes (Hem).

To assess the floristic similarity among the three inselbergs, was prepared a matrix of binary data (presence/absence) compiled from the occurrence of epiphytes in 111 sampled phorophytes. We used the unweighted pair group method with arithmetic mean (UPGMA), incorporating Jaccard's coefficient as a measure of distance, with the aid of software Paleontological Statistics - PAST v. 1.89 (Hammer et al. 2001). Species not identified to species level (sp.) were excluded and species with dubious identification (order [aff.]) were considered.

The species richness was compared with that found in other locations, based on studies that treated similar numbers of phorophytes (Kersten \& Silva 2001, 2002; Werneck \& Espírito-Santo 2002; Gonçalves \& Waechter 2003; Giongo \& Waechter 2004; Alves et al. 2008; Dettke et al. 2008; Fontoura et al. 2009; Kersten \& Kuniyoshi 2009; Blum et al. 2011). The ratios of the numbers of epiphytes and phorophytes were also obtained.

\section{Results}

One hundred and fifty-one species of vascular epiphytes were recorded on Pseudobombax sp. nov., distributed in 77 genera and 21 families (Tab. 1). Angiosperms contributed 125 species, 65 genera and 15 families, while monilophytes and licophytes were represented by 26 species, 12 genera and six families. Orchidaceae contributed 57 species, followed by Bromeliaceae with 31 . The genera with the highest number of species were Epidendrum (Orchidaceae) with eight species, followed by Tillandsia (Bromeliaceae) and Octomeria (Orchidaceae) each with seven species, and Vriesea (Bromeliaceae) and Peperomia (Piperaceae) with six species each.

Campyloneurum centrobrasilianum Lellinger, Isabelia virginalis Barb. Rodr., Nephrolepis exaltata (L.) Schott, Peperomia itatiaiana Yunck., Rhipsalis cf. crispata (Haw.) Pfeiff. and Vanhouttea leonii Chautems are new records for the state of Espírito Santo. 
Table 1 - Vascular epiphytes on Pseudobombax sp. nov. from granitic-gneissic inselbergs in Atlantic Forest, southern Espírito Santo state, Brazil. Locality: PL = Pedra Lisa (submontane inselberg); PP = Pedra dos Pontões (montane inselberg); $\mathrm{PR}=$ Pedra Roxa (upper montane inselberg); $\mathrm{Cat}=$ Ecological category $($ Epi = characteristic holoepiphytes; $\mathrm{Fac}=$ facultative holoepiphyte; $\mathrm{Aci}=$ accidental holoepiphytes; $\mathrm{Hem}=$ hemiepiphyte $).$ Collectors: $\mathrm{DRC}=$ Dayvid Rodrigues Couto; LK= Ludovic Kollmann.

Family (number species) / Species

AMARYLLIDACEAE (1)

Hippeastrum aulicum (Ker Gawl.) Herb.

ANEMIACEAE (3)

Anemia aspera (Feé) Baker

Anemia tomentosa var. anthriscifolia (Schard.) Mickel

Anemia villosa Humb. \& Bonpl. ex Willd.

ARACEAE (6)

Anthurium scandens (Aubl.) Engl.

Anthurium solitarium Schott

Anthurium sp.1

Anthurium sp.2

Philodendron cordatum Kunth ex Schott

Philodendron edmundoi G.M.Barroso

ARALIACEAE (1)

Oreopanax capitatus (Jacq.) Decne. \& Planch.

ASPLENIACEAE (3)

Asplenium auritum $\mathrm{Sw}$.

Asplenium harpeodes Kunze

Asplenium praemorsum Sw.

ASTERACEAE (2)

Cyrtocymura scorpioides (Lam.) H.Rob.

Eremanthus crotonoides (DC.) Sch.Bip.

BEGONIACEAE (3)

Begonia angularis Raddi

Begonia curtii L.B.Sm. \& B.G.Schub.

Begonia reniformis Dryand.

BROMELIACEAE (31)

Aechmea nudicaulis (L.) Griseb.

Aechmea ramosa Mart. ex Schult. \& Schult.f.

Aechmea squarrosa Baker

Alcantarea extensa (L.B.Sm.) J.R.Grant

Alcantarea patriae Versieux \& Wand.

Billbergia horrida Regel

\begin{tabular}{ccc}
\multicolumn{3}{c}{ Locality } \\
PL & PP & PR
\end{tabular} Voucher

$\mathrm{X} \quad \mathrm{Fac}$

DRC 2245 (VIES)

X

$\mathrm{X}$

Aci

Aci

X Aci

DRC 2209 (VIES)

DRC 2153 (VIES)

DRC 2262 (VIES)

$\begin{array}{llll}\text { X } & \text { X } & \text { Epi } & \text { DRC 214 (MBML) } \\ \text { X } & & \text { Fac } & \text { DRC 1323 (VIES) } \\ \text { X } & & \text { Aci } & \text { DRC 2103 (VIES) } \\ \text { X } & \text { X } & \text { Epi } & \text { DRC 2105 (VIES) } \\ \text { X } & & \text { Hem } & \text { DRC 1347 (VIES) } \\ \text { X } & \text { X } & \text { Aci } & \text { DRC 1556 (VIES) }\end{array}$

X

Hem

DRC 2072 (VIES)

X

Epi

DRC 1452 (VIES)

$\mathrm{X} \quad$ Epi DRC 2120(VIES)

X

Epi

DRC 2230 (VIES)

$$
\text { X }
$$

Aci

DRC 1390 (VIES)

X

Aci

DRC 1375 (VIES)

$\begin{array}{llll} & \text { X } & \text { Aci } & \text { DRC 2246 (VIES) } \\ X & & \text { Aci } & \text { LK 6793 (MBML) } \\ \text { X } & \text { X } & \text { Aci } & \text { DRC 2244 (VIES) }\end{array}$

X X

X

Epi

DRC 208 (MBML)

$X \quad$ Fac

DRC 180 (MBML)

$\mathrm{X}$

$\mathrm{Fac}$

DRC 1457 (VIES)

X

Aci

DRC 1428 (VIES)

X X

Aci

DRC 2153 (vies)

Fac

DRC 175 (MBML) 


\begin{tabular}{|c|c|c|c|c|c|}
\hline \multirow{2}{*}{ Family (number species) / Species } & \multicolumn{3}{|c|}{ Locality } & \multirow{2}{*}{ Cat } & \multirow{2}{*}{ Voucher } \\
\hline & PL & PP & PR & & \\
\hline Billbergia tweedieana Baker & & $\mathrm{X}$ & & Epi & DRC 1346 (VIES) \\
\hline Billbergia zebrina (Herb.) Lindl. & & $\mathrm{X}$ & & Epi & DRC 2111 (VIES) \\
\hline Catopsis sessiliflora (Ruiz \& Pav.) Mez & & $\mathrm{X}$ & & Epi & DRC 1350 (VIES) \\
\hline Edmundoa lindenii (Regel) Leme & & $\mathrm{X}$ & & Fac & DRC 1456 (VIES) \\
\hline Neoregelia dayvidiana Leme \& A.P.Fontana & & $\mathrm{X}$ & & Epi & LK 7566 (MBML) \\
\hline Neoregelia pauciflora L.B.Sm. & & $\mathrm{X}$ & & Fac & DRC 2106 (MBML) \\
\hline Neoregelia sp. & & & $\mathrm{X}$ & Epi & DRC 2253 (VIES) \\
\hline Pitcairnia abyssicola Leme \& Kollmann & & $\mathrm{X}$ & & Aci & LK 7873 (MBML) \\
\hline Pitcairnia flammea Lindl. & & & $\mathrm{X}$ & Aci & DRC 1451 (VIES) \\
\hline Quesnelia arvensis (Vell.) Mez & & $\mathrm{X}$ & & Aci & DRC 2068 (VIES) \\
\hline Quesnelia kautskyi C.M.Vieira & & $\mathrm{X}$ & & Aci & DRC 276 (MBML) \\
\hline Quesnelia strobilispica Wawra & & $\mathrm{X}$ & & Fac & DRC 174 (MBML) \\
\hline Tillandsia gardneri Lindl. & $\mathrm{X}$ & $\mathrm{X}$ & & Epi & DRC 186 (MBML) \\
\hline Tillandsia geminiflora Brongn. & & & $\mathrm{X}$ & Epi & DRC 2228 (VIES) \\
\hline Tillandsia loliacea Mart. ex Schult. \& Schult.f. & $\mathrm{X}$ & & & Epi & DRC 2137 (VIES) \\
\hline Tillandsia recurvata $(\mathrm{L}.) \mathrm{L}$. & & $\mathrm{X}$ & $\mathrm{X}$ & Epi & DRC 2126 (VIES) \\
\hline Tillandsia stricta Sol. & $\mathrm{X}$ & $\mathrm{X}$ & $\mathrm{X}$ & Epi & DRC 2222 (VIES) \\
\hline Tillandsia tenuifolia $\mathrm{L}$. & & $\mathrm{X}$ & & Epi & DRC 2110 (VIES) \\
\hline Tillandsia usneoides (L.) L. & & $\mathrm{X}$ & $\mathrm{X}$ & Epi & DRC 2223 (VIES) \\
\hline Vriesea capixabae Leme & & & $\mathrm{X}$ & Epi & not collected \\
\hline Vriesea gigantea Gaudich. & & $\mathrm{X}$ & & Epi & DRC 2128 (VIES) \\
\hline Vriesea lubbersii (Baker) E.Morren & & $\mathrm{X}$ & $\mathrm{X}$ & Epi & DRC 242 (MBML) \\
\hline Vriesea poenulata (Baker) E.Morren ex Mez & & & $\mathrm{X}$ & Epi & DRC 2257 (VIES) \\
\hline Vriesea aff. procera (Mart. ex Schult. \& Schult.f.) Wittm. & $\mathrm{X}$ & & & Epi & DRC 2138 (R) \\
\hline Vriesea vagans (L.B.Sm.) L.B.Sm. & & $\mathrm{X}$ & $\mathrm{X}$ & Epi & DRC 1857 (VIES) \\
\hline \multicolumn{6}{|l|}{ CACTACEAE (8) } \\
\hline Epiphyllum phyllanthus (L.) Haw. & $\mathrm{X}$ & & & Epi & not collected \\
\hline Hatiora salicornioides (Haw.) Britton \& Rose & & $\mathrm{X}$ & & Epi & DRC 2081 (MBML) \\
\hline Hylocereus setaceus (Salm-Dyck) R.Bauer & $\mathrm{X}$ & & & Epi & DRC 2219 (VIES) \\
\hline Lepismium cruciforme (Vell.) Miq. & & $\mathrm{X}$ & & Epi & LK 6804 (MBML) \\
\hline Rhipsalis crispata (Haw.) Pfeiff. & & $\mathrm{X}$ & & Epi & LK 6803 (MBML) \\
\hline Rhipsalis lindbergiana K.Schum. & & $\mathrm{X}$ & $\mathrm{X}$ & Epi & DRC 2121 (VIES) \\
\hline Rhipsalis neves-armondii K. Schum & & $\mathrm{X}$ & & Epi & DRC 1952 (VIES) \\
\hline Rhipsalis teres (Vell.) Steudt. & & $\mathrm{X}$ & $\mathrm{X}$ & Epi & DRC 1238 (VIES) \\
\hline \multicolumn{6}{|l|}{ CLUSIACEAE (1) } \\
\hline Clusia aemygdioi Gomes da Silva \& B.Weinberg & & $\mathrm{X}$ & & Hem & DRC 2073 (VIES) \\
\hline GESNERIACEAE (5) & & & & & \\
\hline
\end{tabular}


Family (number species) / Species

Nematanthus hirtellus (Schott) Wiehler

Paliavana prasinata (Ker Gawl.) Benth.

Sinningia magnifica (Otto \& A.Dietr.) Wiehler

Sinningia speciosa (Lodd.) Hiern

Vanhouttea leonii Chautems

LOMARIOPSIDACEAE (1)

Nephrolepis exaltata (L.) Schott

MELASTOMATACEAE (1)

Tibouchina heteromalla (D.Don) Cogn.

MORACEAE (1)

Ficus arpazusa Casar.

\section{ORCHIDACEAE (57)}

Acianthera auriculata (Lindl.) Pridgeon \& M.W.Chase Acianthera crinita (Barb.Rodr.) Pridgeon \& M.W.Chase Acianthera heliconiscapa (Hoehne) F.Barros

Acianthera leptotifolia (Barb.Rodr.) Pridgeon \& M.W.Chase Acianthera luteola (Lindl.) Pridgeon \& M.W.Chase Acianthera saurocephala (Lodd.) Pridgeon \& M.W.Chase Aspasia lunata Lindl.

Barbosella spiritusanctensis (Pabst) F.Barros \& Toscano

Brasilidium crispum (Lodd.) Campacci

Brasiliorchis marginata (Lindl.) R.B.Singer et al.

Brasiliorchis phoenicanthera (Barb.Rodr.) R.B.Singer et al.

Brasiliorchis picta (Hook.) R.B.Singer et al.

Bulbophyllum cantagallense (Barb.Rodr.) Cogn.

Bulbophyllum micropetaliforme J.E.Leite

Bulbophyllum sp.

Christensonella pachyphylla (Schltr. ex Hoehne) Szlach. et al.

Christensonella subulata (Lindl.) Szlach. et al.

Cyclopogon argyrifolius Barb.Rodr.

Cyrtopodium glutiniferum Raddi

*Dendrobium nobile Lindl.

Elleanthus brasiliensis (Lindl.) Rchb.f.

Encyclia patens Hook.

Epidendrum avicula Lindl.

Epidendrum campaccii Hágsater \& L.Sánchez

Epidendrum densiflorum Hook.

\begin{tabular}{|c|c|c|c|c|}
\hline \multicolumn{3}{|c|}{ Locality } & \multirow{2}{*}{ Cat } & \multirow{2}{*}{ Voucher } \\
\hline PL & PP & PR & & \\
\hline & $\mathrm{X}$ & & Epi & DRC 1334 (VIES) \\
\hline & $\mathrm{X}$ & & Aci & DRC 311 (MBML) \\
\hline \multirow{3}{*}{$X$} & & $\mathrm{X}$ & Fac & DRC 2226 (VIES) \\
\hline & & & Aci & DRC 2207 (VIES) \\
\hline & & $\mathrm{X}$ & Aci & DRC 2247 (VIES) \\
\hline
\end{tabular}

X $\quad$ Fac $\quad$ DRC 1348 (VIES)

X

Aci DRC 1374 (VIES)

X Hem DRC 1406(VIES)

$\mathrm{X} \quad \mathrm{X}$ Epi DRC 1385(VIES)

$\mathrm{X} \quad$ Epi DRC 91 (MBML)

X Epi DRC 324 (MBML)

X Epi DRC 2096 (MBML)

X Epi DRC 268 (MBML)

X Epi DRC 172 (MBML)

X Epi DRC 1328(VIES)

X Epi DRC 618 (MBML)

$\mathrm{X} \quad \mathrm{X}$ Epi DRC 239 (MBML)

$\mathrm{X} \quad \mathrm{X}$ Epi DRC 147 (MBML)

$\mathrm{X}$ Epi DRC 125 (MBML)

$\mathrm{X}$

Epi DRC 2220(VIES)

$\mathrm{X} \quad \mathrm{X}$ Epi DRC 1449 (VIES)

$\mathrm{X}$ Epi DRC 2276(VIES)

X

Epi DRC 2225

$\mathrm{X} \quad$ Epi DRC 23 (MBML)

$\mathrm{X} \quad \mathrm{X}$ Epi DRC 2252 (VIES)

$\mathrm{X}$ Aci not collected

$\mathrm{X} \quad \mathrm{X}$ Aci DRC 190 (MBML)

$\mathrm{X} \quad$ Epi not collected

X Fac DRC 1400 (VIES)

$\mathrm{X} \quad \mathrm{X}$ Epi DRC 1243 (VIES)

X Epi DRC 2124(VIES)

$\mathrm{X} \quad \mathrm{X}$ Epi DRC 2123 (VIES)

$\mathrm{X} \quad \mathrm{F} \quad$ Dac 2260 (VIES) 
Family (number species) / Species

Epidendrum filicaule Lindl.

Epidendrum rigidum Jacq.

Epidendrum secundum Jacq.

Epidendrum tridactylum Lindl.

Grandiphyllum divaricatum (Lindl.) Docha Neto

Heterotaxis brasiliensis (Brieger \& Illg) F.Barros

Isabelia virginalis Barb.Rodr.

Isochilus linearis (Jacq.) R.Br.

Laelia gloriosa (Rchb.f.) L.O.Williams

Lankesterella longicollis (Cogn.) Hoehne

Masdevallia infracta Lindl.

Maxillariella robusta (Barb.Rodr.)M.A.Blanco \& Carnevali

Mormolyca rufescens (Lindl.) M.A.Blanco

Octomeria crassifolia Lindl.

Octomeria decumbens Cogn.

Octomeria cf. diaphana Lindl.

Octomeria sp. 1

Octomeria sp. 2

Octomeria sp. 3

Octomeria sp. 4

Promenaea xanthina (Lindl.) Lindl.

Ornithidium rigidum (Barb.Rodr.) M.A.Blanco \& Ojeda

Polystachya concreta (Jacq.) Garay \& Sweet

Prescottia plantaginifolia Lindl. ex Hook.

Prosthechea cf. bulbosa (Vell.) W.E.Higgins

Prosthechea calamaria (Lindl.) W.E.Higgins

Prosthechea fragrans (Sw.) W.E.Higgins

Sophronitis cernua Lindl.

Specklinia grobyi (Batem. ex Lindl.) F.Barros

Stelis argentata Lindl.

Xylobium variegatum (Ruiz \& Pav.) Garay \& Dunst.

Zygopetalum intermedium Lodd.

PIPERACEAE (6)

Peperomia alata Ruiz \& Pav.

Peperomia glabella (Sw.) A.Dietr. var. glabella

Peperomia itatiaiana Yunck.

Peperomia rubricaulis (Nees) A.Dietr.

\begin{tabular}{|c|c|c|c|c|}
\hline \multicolumn{3}{|c|}{ Locality } & \multirow{2}{*}{ Cat } & \multirow{2}{*}{ Voucher } \\
\hline PL & PP & PR & & \\
\hline \multirow[t]{8}{*}{$\mathrm{X}$} & & & Epi & DRC 2149 (VIES) \\
\hline & $X$ & & Epi & DRC 2122 (VIES) \\
\hline & & $\mathrm{X}$ & Aci & DRC 2083 (MBML) \\
\hline & $\mathrm{X}$ & & Epi & DRC 90 (MBML) \\
\hline & & $\mathrm{X}$ & Epi & not collected \\
\hline & $\mathrm{X}$ & $\mathrm{X}$ & Epi & DRC 76 (MBML) \\
\hline & & $\mathrm{X}$ & Epi & DRC 2234 (VIES) \\
\hline & $\mathrm{X}$ & $\mathrm{X}$ & Epi & DRC 2236 (VIES) \\
\hline \multirow[t]{3}{*}{$\mathrm{X}$} & & & Epi & DRC 2208 (VIES) \\
\hline & $\mathrm{X}$ & & Epi & DRC 1404 (VIES) \\
\hline & & $\mathrm{X}$ & Epi & DRC 2275 (VIES) \\
\hline \multirow[t]{11}{*}{$\mathrm{X}$} & & & Epi & DRC 320 (MBML) \\
\hline & $\mathrm{X}$ & $\mathrm{X}$ & Epi & DRC 30 (MBML) \\
\hline & $\mathrm{X}$ & $\mathrm{X}$ & Epi & DRC 2240 (VIES) \\
\hline & $\mathrm{X}$ & & Epi & DRC 325 (MBML) \\
\hline & & $\mathrm{X}$ & Epi & DRC 2256 \\
\hline & & $\mathrm{X}$ & Epi & DRC 2237 (VIES) \\
\hline & $\mathrm{X}$ & & Epi & DRC 297 (MBML) \\
\hline & $\mathrm{X}$ & & Epi & DRC 2115 (VIES) \\
\hline & $\mathrm{X}$ & & Epi & DRC 2132 (VIES) \\
\hline & & $\mathrm{X}$ & Epi & DRC 2235 (VIES) \\
\hline & $\mathrm{X}$ & & Epi & DRC 234 (MBML) \\
\hline \multirow[t]{4}{*}{$\mathrm{X}$} & $\mathrm{X}$ & $\mathrm{X}$ & Epi & DRC 2216 (VIES) \\
\hline & $\mathrm{X}$ & & Aci & DRC 140 (MBML) \\
\hline & $\mathrm{X}$ & $\mathrm{X}$ & Epi & DRC 2259 \\
\hline & & $\mathrm{X}$ & Epi & DRC 2268 \\
\hline $\mathrm{X}$ & & & Epi & DRC 2217 (VIES) \\
\hline \multirow[t]{5}{*}{$\mathrm{X}$} & & & Epi & DRC 2148 (VIES) \\
\hline & $\mathrm{X}$ & $\mathrm{X}$ & Epi & DRC 139 (MBML) \\
\hline & $\mathrm{X}$ & $\mathrm{X}$ & Epi & DRC 2079 (MBML) \\
\hline & $\mathrm{X}$ & & Aci & DRC 189 (MBML) \\
\hline & X & & Aci & DRC 254 (MBML) \\
\hline
\end{tabular}

X Fac

DRC 2284 (VIES)

DRC 2301 (VIES)

DRC 2102 (VIES)

X

Aci

DRC 1380 (VIES) 
Family (number species) / Species

Peperomia tetraphylla (G.Forst.) Hook. \& Arn.

Peperomia trinervis Ruiz \& Pav. var. trinervis

POACEAE (1)

**Melinis minutiflora P.Beauv.

POLYPODIACEAE (14)

Campyloneurum acrocarpon Fée

Campyloneurum centrobrasilianum Lellinger

Microgramma percussa (Cav.) de la Sota

Microgramma squamulosa (Kaulf.) de la Sota

Microgramma tecta (Kaulf.) Alston

Niphidium crassifolium (L.) Lellinger

Pecluma pectinatiformis (Lindm.) M.G.Price

Pecluma plumula (Willd.) M.G.Price

Phlebodium aureum (L.) J.Sm.

Pleopeltis astrolepis (Liebm.) E.Fourn.

Pleopeltis hirsutissima (Raddi) de la Sota

Pleopeltis minima (Bory) J. Prado \& R.Y. Hirai

Pleopeltis monoides (Weath.) Salino

Pleopeltis pleopeltifolia (Raddi) Alston

PTERIDACEAE (4)

Doryopteris collina (Raddi) J. Sm.

Doryopteris magdalenensis (Brade) Brade

Doryopteris sp.

Hemionitis tomentosa (Lam.) Raddi

SELAGINELLACEAE (1)

Selaginella convoluta (Arn.) Spring

SOLANACEAE (1)

Markea atlantica Stehmann \& Giacomin

TOTAL

(*) casual alien species; $(* *)$ invasive species
In addition, two species, Neoregelia dayvidiana Leme \& A.P.Fontana and Pitcairnia abyssicola Leme \& L.Kollmann (Bromeliaceae) are endemic to the Pedra dos Pontões region; and two species, Dendrobium nobile Lindl. (Orchidaceae) and Melinis minutiflora P.Beauv. (Poaceae), are exotic.

\begin{tabular}{|c|c|c|c|c|}
\hline \multicolumn{3}{|c|}{ Locality } & \multirow{2}{*}{ Cat } & \multirow{2}{*}{ Voucher } \\
\hline PL & PP & PR & & \\
\hline & $\mathrm{X}$ & & Epi & DRC 2097 (MBML) \\
\hline & $\mathrm{X}$ & & Fac & DRC 1237 (VIES) \\
\hline & $\mathrm{X}$ & $\mathrm{X}$ & Aci & DRC 2231 (VIES) \\
\hline & $\mathrm{X}$ & & Aci & DRC 1331 (VIES) \\
\hline & $\mathrm{X}$ & $\mathrm{X}$ & Epi & DRC 2254 (VIES) \\
\hline & $\mathrm{X}$ & & Epi & DRC 1445 (VIES) \\
\hline & $\mathrm{X}$ & $\mathrm{X}$ & Epi & DRC 2270 (VIES) \\
\hline & $\mathrm{X}$ & & Epi & DRC 2116 (VIES) \\
\hline & $\mathrm{X}$ & $\mathrm{X}$ & Epi & DRC 2109 (VIES) \\
\hline & $\mathrm{X}$ & & Epi & DRC 2118 (VIES) \\
\hline \multirow[t]{4}{*}{$\mathrm{X}$} & & & Epi & DRC 2221 (VIES) \\
\hline & & $\mathrm{X}$ & Aci & DRC 2271 (VIES) \\
\hline & $\mathrm{X}$ & & Epi & DRC 2108 (VIES) \\
\hline & $\mathrm{X}$ & & Epi & DRC 2088 (MBML) \\
\hline \multirow[t]{6}{*}{$X$} & $\mathrm{X}$ & & Epi & DRC 2127 (MBML) \\
\hline & & $\mathrm{X}$ & Epi & DRC 2270 (VIES) \\
\hline & $\mathrm{X}$ & & Epi & DRC 1954 (VIES) \\
\hline & $\mathrm{X}$ & & Aci & DRC 1955 (VIES) \\
\hline & & $\mathrm{X}$ & Aci & DRC 2261 (VIES) \\
\hline & $\mathrm{X}$ & & Aci & DRC 1558 (VIES) \\
\hline $\mathrm{X}$ & & & Aci & DRC 2210 (VIES) \\
\hline $\mathrm{X}$ & & $\mathrm{X}$ & Aci & DRC 2152 (VIES) \\
\hline & $X$ & & Epi & DRC 1455 (VIES) \\
\hline 24 & 105 & 62 & & \\
\hline
\end{tabular}

Of the total number of species, 24 were recorded for Submontane Inselberg (PL), 105 for Montane Inselberg (PP) and 62 for Upper montane Inselberg (PR). Orchidaceae (57 species) was the richest family in all areas, followed by Bromeliaceae (26 species) (Fig. 3). Polypodiaceae ranked third 
in PP and PR, whereas Cactaceae ranked third in PL. Members of Bromeliaceae, Cactaceae, Gesneriaceae, Orchidaceae, Polypodiaceae and Pteridaceae were common to all three inselbergs. Of the 77 genera found, 10 were common to all three areas (Aechmea, Alcantarea, Tillandsia, Vriesea, Brasiliorchis, Bulbophyllum, Epidendrum, Polystachya, Prosthechea and Pleopeltis), while 21 were unique to PP, nine to PR and six to PL.

Overall, considering the 111 sampled phorophytes, we recorded 151 species of vascular epiphytes, while non-parametric estimators indicated asymptotic richness between 159.0 (ICE) and 166.8 (Jachnife 1), suggesting that few species inventoried would be expanded if the sampling indicate that 90 and $95 \%$ of species richness of

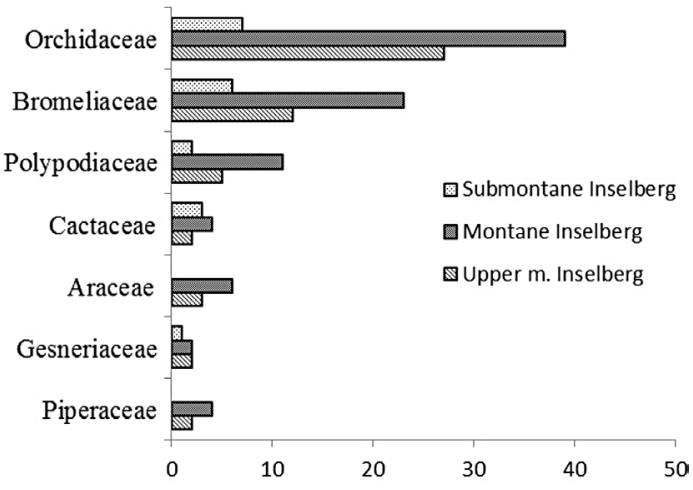

Figure 3 - Species richness of the most important families of epiphytes on Pseudobombax sp. nov. from three granite-gneiss inselbergs in Atlantic Rainforest, southern Espírito Santo state, Brazil.
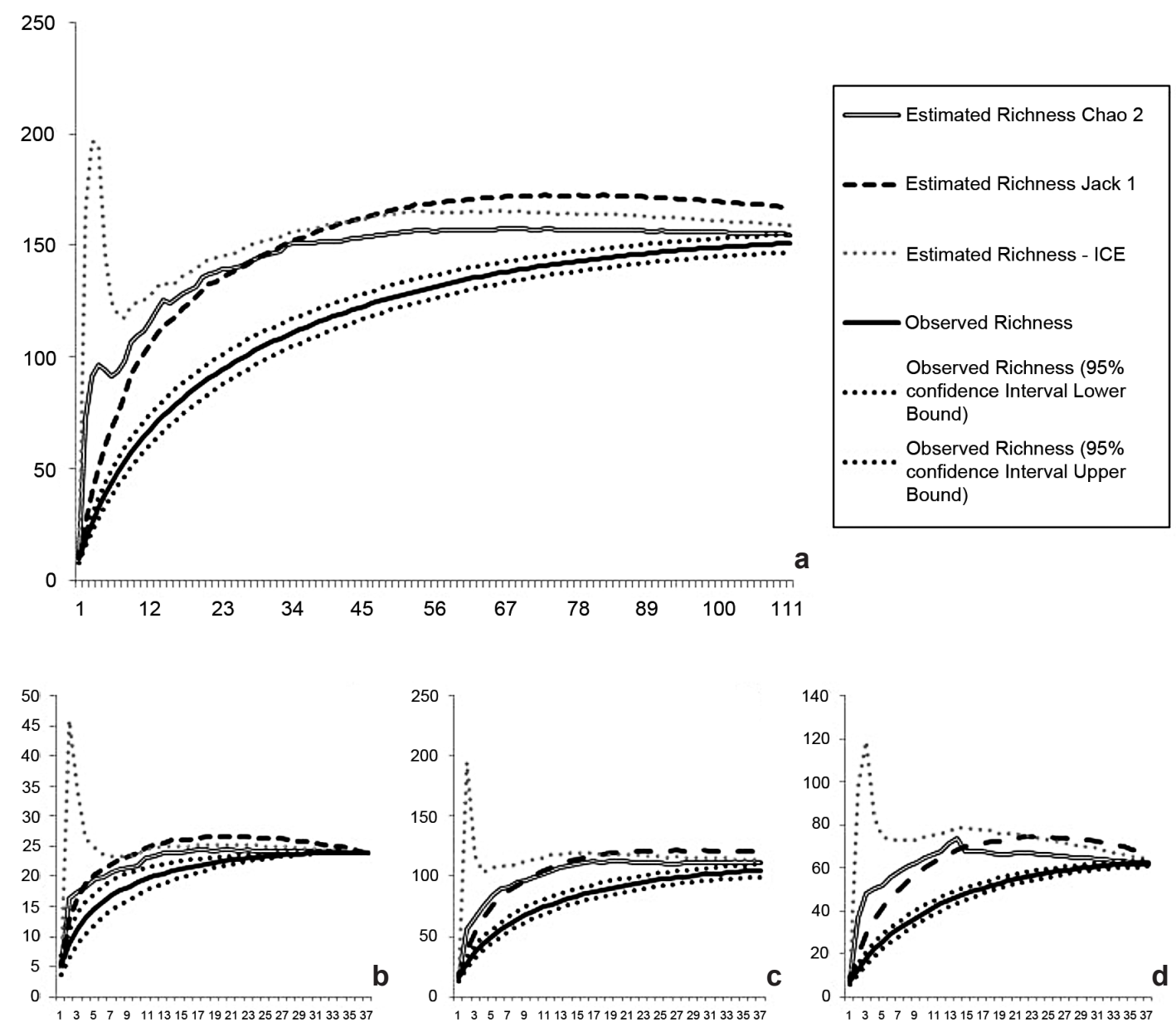

Figure 4-Rarefaction curve and richness estimations for the 111 phorophytes sampled on Atlantic Rainforest inselbergs (a) and the three inselbergs sampled: Submontane inselberg (b), Montane inselberg (c) and Upper montane inselberg (d) showing the observed number of species in our dataset (black solid line), its 95\% confidence interval (dotted black lines) and the number of species estimated for the three inselbergs, using three usual richness estimators: ICE (incidence-based coverage estimator), Chao 2 and Jackknife 1. 
epiphytes was recorded. Analyzing the three inselbergs separately, it is observed that in PL, the sampled epiphytic community in this study was an amount equal to the estimated values (Chao 2, ICE and Jackknife 1), already in PP and PR can be found new occurrences epiphytic species. In PP is estimated that $87 \%$ (120, Jackknife 1) and 91\% (114, ICE), while in PR 93\% (64, ICE) and 96\% (66, Jackknife 1) the total specific richness was inventoried (Fig. 4).

The analysis of the floristic relationships among the three study areas, calculated using the Jaccard coefficient (Fig. 5) and represented in the Venn diagram (Fig. 6) demonstrated the existence of three distinct floras. PR and PP, both within montane forests, were most similar to each other, with 30 shared species. The most distinct was PL, within a lower montane semideciduous forest, with only three species shared with PP: Alcantarea patriae Versieux \& Wand., Tillandsia

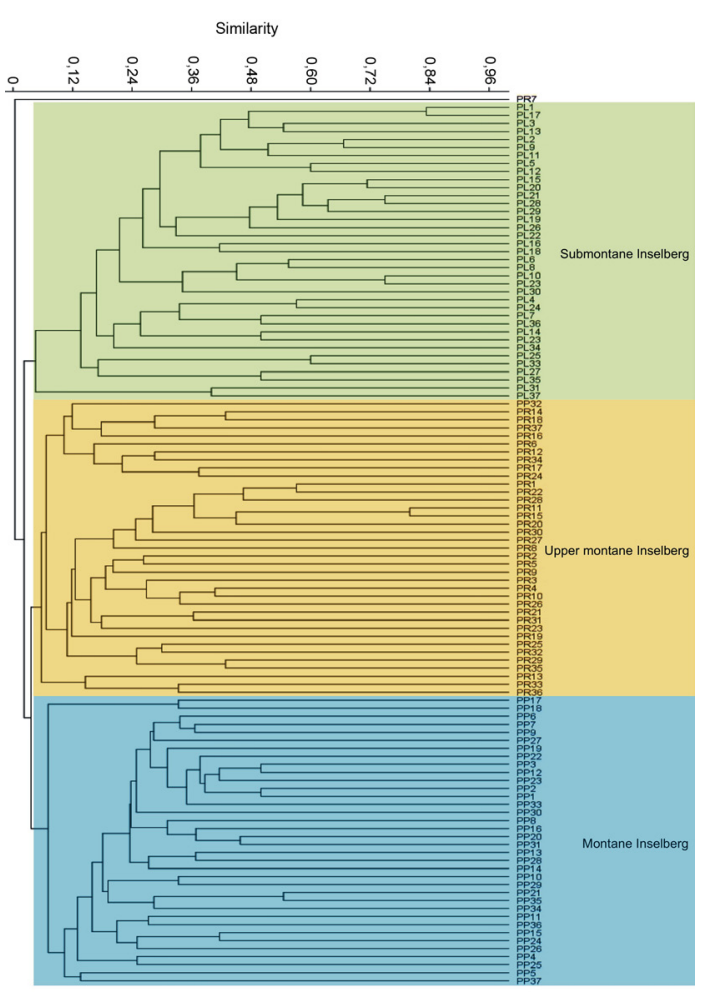

Figure 5 - Dendrogram of floristic similarity (Jaccard, binary data) of vascular epiphytes on 111 individuals of Pseudobombax sp. nov. from three granitic-gneissic inserbergs inventoried in Atlantic Rainforest, southern Espírito Santo state, Brazil. PP = montane inselberg; PR $=$ upper montane inselberg; $\mathrm{PL}=$ submontane inselberg. gardneri Lindl. and Pleopeltis minima (Bory) J.Prado \& R.Y. Hirai. Alcantarea patriae is endemic to inselbergs in southern Espírito Santo, and the other two are widely distributed in South America. PL and PR shared only one species, Selaginella convoluta (Arn.) Spring, which is widely distributed on rocky outcrops in Brazil and South America as a whole. Only three species were shared among all three areas: Aechmea nudicaulis (L.) Griseb., Tillandsia stricta Sol. and Polystachya concreta (Jacq.) Garay \& Sweet.

Of the total of 151 species, $64 \%$ were classified as characteristic holoepiphytes, including all species of Aspleniaceae, Cactaceae and Solanaceae; $23 \%$ as accidental holoepiphytes, represented by all types of families: Anemiaceae, Asteraceae, Begoniaceae, Pteridaceae, Selaginellaceae and Melastomataceae; $10 \%$ as facultative holoepiphytes; and $3 \%$ as hemiepiphytes, including the primary hemiepiphytes Clusia aemygdioi Gomes da Silva \& Weinberg, Ficus arpazusa Casar and Oreopanax capitatus (Jacq.) Decne. \& Planch. and the secondary hemiepiphyte Philodendron cordatum Kunth ex Schott.

Among the species registered, 19 are present on official lists of endangered species. Eight species are under some degree of threat according to the list of endangered flora of Brazil. Four species are listed as "Vulnerable": Begonia curtii L.B.Sm. \& B.G.Schub., Quesnelia kautskyi C.M.Vieira, Grandiphyllum divaricatum (Lindl.) Docha Neto and Isabelia virginalis Barb.Rodr.; and four as "Endangered": Clusia aemygdioi, Vanhouttea leonii Chautems, Peperomia itatiaiana Yunck. and Pleopeltis monoides (Weath.) Salino. Among the species found, 14 species are threatened according to the list of endangered flora of Espírito Santo: Eight species are listed as "Vulnerable": Begonia angularis Raddi, Begonia curtii, Quesnelia kautskyi, Sinningia magnifica (Otto \& A.Dietr.) Wiehler, Sinningia speciosa (Lodd.) Hiern, Brasilidium crispum (Lodd.) Campacci, Brasiliorchis phoenicanthera (Barb.Rodr.) R.B.Singer et al. and Pleopeltis monoides; three as "Endangered": Nematanthus hirtellus (Schott) Wiehler, Acianthera crinita (Barb.Rodr.) Pridgeon \& M.W.Chase and $A$. saurocephala (Lodd.) Pridgeon \& M.W.Chase; and three as "Critically Endangered": Barbosella spiritusanctensis (Pabst) F.Barros \& Toscano, Bulbophyllum cantagallense (Barb.Rodr.) Cogn. and Epidendrum tridactylum Lindl.. 


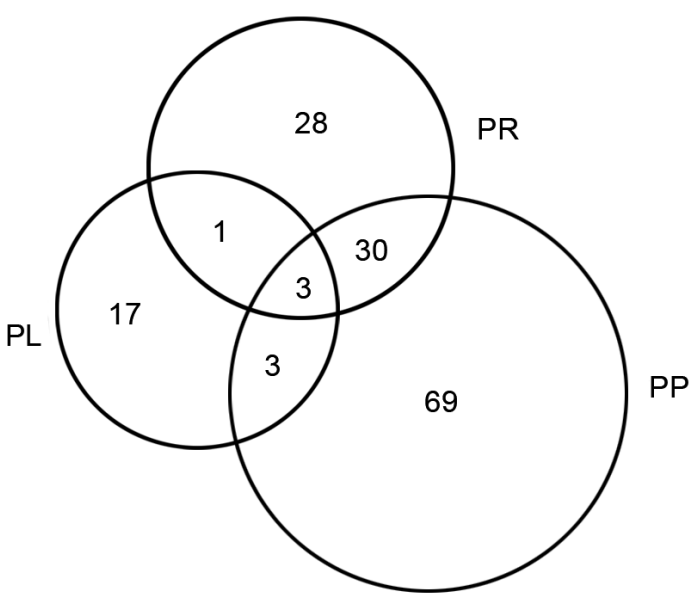

Figure 6 - Venn diagram showing exclusive and shared species among the three study areas: $\mathrm{PL}=$ Submontane inselberg; PP = Montane inselberg; and PR = Upper montane inselberg, Espírito Santo state, Brazil.

\section{Discussion}

The present study provides the first contributions to the knowledge of vascular epiphytic flora on inselbergs. We assessed the vascular epiphytic richness associated with the phorophyte Pseudobombax sp. nov. on three inselbergs in the Brazilian Atlantic Rainforest. Non-parametric estimators (Chao 2, Jackknife 1) indicate that 90 and $95 \%$ of species richness of epiphytes was recorded. The ratio of number of vascular epiphytes and number of phorophytes sampled on Pseudobombax sp. nov., in a montane inselberg, was higher than the richness of vascular epiphytes found on quartzite rocky outcrops located in the Cerrado Domain, and in general, in different forest types of the Atlantic Domain, but smaller in riches for some areas of rain forest of southern Brazil. The inselbergs differ in their floristic composition, although members of Orchidaceae and Bromeliaceae predominate. The inselbergs even being inserted in a completely different vegetation surrounding matrix do not represent a barrier for distribution of vascular epiphytes.

These families of epiphytic flora are also among the most speciose in Brazil (Kersten \& Silva 2001, 2002; Giongo \& Waechter 2004; Kersten \& Kuniyoshi 2009; Blum et al. 2011) and also in the extra-tropical regions of the country (Küper et al. 2004; Krömer et al. 2005, 2007). In the present study, Orchidaceae and Bromeliaceae contributed $55 \%$ of the total number of species recorded on
Pseudobombax sp. nov., a similar percentage to that observed by other studies (Geraldino et al. 2010; Blum et al. 2011).

The high species richness of epiphytes is an unusual aspect of the types of rocky vegetation such as on inselbergs, where extreme environmental factors (e.g., total or partial absence of soil and nutrients, water scarcity, and direct wind exposure) limit the establishment and longevity of larger trees that support a greater richness of epiphytes (Hernández-Rosas \& Carlsen 2003; Woods et al. 2014). The high richness of epiphytes on the inselbergs studied here (about 151 species on 111 phorophytes) may be related to the size and architecture of Pseudobombax sp. nov. These trees have thick, often horizontal branches, can reach a height of $15 \mathrm{~m}$ and a DBH over $100 \mathrm{~cm}$, and have thick, exposed roots on the rocky surface, thus forming an important site for epiphyte colonization.

For a precise comparison of the richness of epiphytes in different vegetation types, we used only studies in which the number of phorophytes sampled was provided (see Methods). The richness of epiphytes recorded on the inselbergs studied here was higher than the richness of epiphytes found on quartzite rocky outcrops in the Cerrado and different subtypes of the Atlantic Forest, except in Dense Ombrophilous Forest and Anthropic Vegetation, both located in southern Brazil (Tab. 2 ). In comparison with rocky physiognomies in the Cerrado, our data showed that the highest richness occurred either when epiphytes of a specific host tree, for example, Vellozia piresiana L.B.Sm. (Werneck \& Espírito-Santo 2002), were considered, or when the epiphyte flora associated with different species of phorophytes was considered (Alves et al. 2008). In both cases, the factor that contributed most to the lower species richness in these environments seems to be related to the Cerrado Domain, which supports a lower epiphytic richness than is found in the Atlantic Domain (Bataghin et al. 2012). However, trunks of Vellozia are colonised by specific epiphytes of inselbergs and rocky fields (Porembski 2005), supporting an endemic epiphytic flora of rupestrian ecosystems of Orchidaceae (e.g., Constantia cipoensis Porto \& Brade; Leptotes vellozicola van den Berg et al.; Grobya cipoensis F.Barros \& Lourenço, Lepanthopsis vellozicola R.C. Mota et al.) as well as most of the 12 species of genus Pseudolaelia (Menini Neto et al. 2013) and the bromeliad Vriesea vellozicola Leme \& J.A. Siqueira. On the other hand Pseudobombax sp. 
Table 2 - Studies performed on vascular epiphytes in different vegetation formations in the Atlantic Forest and Cerrado domains in Brazil, the number of phorophytes sampled, richness of epiphytes, and ratio of epiphytes to phorophytes (Nspe = number of epiphytic species; NfA = number of sampled phorophytes), ordered by this last column. (Physiognomy: DOF = Dense Ombrophilous Forest; MOF = Mixed Ombrophilous Forest; SSF = Seasonal Semideciduous Forest; GF = Gallery Forest; RF = Restinga Forest; SMI = Submontane Inselberg; MI = Montane Inselberg; UMI = Upper Montane Inselberg; QRO = Quartzite Rocky Outcrops; Antro. = Anthropic Vegetation).

\begin{tabular}{|c|c|c|c|c|c|c|}
\hline Referece & $\begin{array}{l}\text { Phytogeographic } \\
\text { Domain }\end{array}$ & Physiognomy & Phorophyte type & $\begin{array}{l}\text { Number } \\
\text { phorophyte } \\
\text { sampled }\end{array}$ & $\begin{array}{l}\text { Richness of } \\
\text { epiphytes }\end{array}$ & $\begin{array}{l}\text { Ratio } \\
\text { Nspe/ NfA }\end{array}$ \\
\hline This study & Atlantic Forest & MI & Pseudobombax sp. nov. & 37 & 89 & 2,4 \\
\hline Blum et al. 2011 & Atlantic Forest & DOF & Several & 120 & 278 & 2,3 \\
\hline This study & Atlantic Forest & UMI & Pseudobombax sp. nov. & 37 & 51 & 1,4 \\
\hline Gonçalves \& Waechter 2003 & Atlantic Forest & Antro. & Ficus organensis & 60 & 77 & 1,3 \\
\hline Kersten \& Kuniyoshi 2009 & Atlantic Forest & MOF & Several & 100 & 114 & 1,1 \\
\hline Alves et al. 2008 & Cerrado & QRO & Several & 56 & 53 & 0,9 \\
\hline Giongo \& Waechter 2004 & Atlantic Forest & GF & Several & 60 & 50 & 0,8 \\
\hline Kersten \& Silva 2001 & Atlantic Forest & $\mathrm{RF}$ & Several & 110 & 77 & 0,7 \\
\hline This study & Atlantic Forest & SMI & Pseudobombax sp. nov. & 37 & 19 & 0,5 \\
\hline Kersten \& Silva 2002 & Atlantic Forest & SSF & Several & 110 & 49 & 0,4 \\
\hline Fontoura et al. 2009 & Atlantic Forest & $\mathrm{RF}$ & Several & 110 & 34 & 0,3 \\
\hline Dettke et al. 2008 & Atlantic Forest & $\mathrm{SSF}$ & Several & 90 & 29 & 0,3 \\
\hline Werneck \& Espírito-Santo 2002 & Cerrado & QRO & Vellozia piresiana & 98 & 6 & 0,1 \\
\hline
\end{tabular}

nov., houses a typical epiphytic flora of the forest ecosystems, although it is an endemic species of inselbergs.

This marked difference in epiphytic composition on these two phorophytes enables understand that Pseudobambax sp.nov. is directly responsible for the homogeneous distribution of the population of epiphytes in the inselbergs of the region, because even being inserted in a completely different vegetation surrounding matrix do not represent a barrier for distribution of species.

The Atlantic Forest Domain, of which these inselbergs are a part, harbors the main centers of diversity and endemism for many typically epiphytic families and genera (Pabst \& Dungs 1975, 1977; Smith \& Downs 1977, 1979; Martinelli et al. 2008). The marked climatic and geomorphological diversity (Rizzini 1997) contributes to the floral richness of this region, which is an important depository of vascular epiphytic flora (Kersten 2010). Comparing the results of this study with those obtained in surveys conducted in forest ecosystems with multiple phorophytes, the epiphytic richness found on
Pseudobombax sp. nov. can be considered high (Tab. 2). This richness is higher than in different forest types in the Atlantic Forest Domain, such as Dense Ombrophilous Forest (Giongo \& Waechter 2004), Mixed Ombrophilous Forest (Kersten \& Silva 2002; Kersten \& Kuniyoshi 2009), Seasonal Semideciduous Forest (Dettke et al. 2008) and Restinga Vegetation Forest (Kersten \& Silva 2001; Fontoura et al. 2009). The high richness found on Pseudobombax sp. nov. appears to be related to the peculiar structure of these morphological phorophytes, which extend large roots over the rock surface, allowing extensive colonization of epiphytic flora.

The vegetation heterogeneity of the matrix surrounding the three sites (Lower Montane, Montane Semideciduous Forest and Dense Ombrophilous Forest) is another factor that contributes to the high richness of epiphytes. This was also observed in other studies conducted in areas with a wide range of vegetation types in ecotone zones with a confluence of forest formations (Kersten \& Kuniyoshi 2009; MeniniNeto et al. 2009; Geraldino et al. 2010), greatly increasing the epiphytic richness. The epiphyte 
richness on inselbergs was lower compared to the Dense Ombrophilous Forest in Paraná (Blum et al. 2011), where 278 species were recorded on 120 phorophytes (Tab. 2); this is the highest epiphytic richness ever recorded in Brazil. According to the authors, this richness was associated with the steep altitudinal gradient, which encompassed two distinct forest types (Montane and Submontane Dense Ombrophilous Forest), where the high humidity with rainfall well distributed throughout the year provided a favorable environment for epiphytes.

In contrast to the many reports on multiple phorophytes, few studies is available for vascular epiphytes richness on particular phorophyte species. Studies conducted in the Neotropics with specific phorophytes have highlighted the importance of some tree species for the maintenance and conservation of epiphytic flora (Freiberg 1996; Werneck \& Espírito-Santo 2002; Gonçalves \& Waechter 2003; Reis \& Fontoura 2009; Obermüller et al. 2012). In our study, the richness of epiphytes on Pseudobombax sp. nov. is high compared both to a study of 60 individuals of Ficus organensis (Miq.) Miq. in the Atlantic Domain (Gonçalves \& Waechter 2003) and to the richness of epiphyte species on 98 individuals of the specialist phorophyte of rocky environments, Vellozia piresiana (Werneck \& Espiríto-Santo 2002). In the first comparison, although the relationship between the numbers of epiphytes/ phorophytes is similar (Tab. 2), the high richness found might be related to the environments where Pseudobombax sp. nov. and Ficus organensis were studied. The inselbergs areas are steep and difficult to access, which preserves the epiphytic flora associated with this host tree; while in the area where Ficus organensis was studied is disturbed and are more exposed to indiscriminate extraction of ornamental species, which depauperates the epiphyte flora. In the second comparison, the small size of individuals of $V$. piresiana (maximum 2 m high), compared with Pseudobombax sp. nov. (maximum of $15.3 \mathrm{~m}$ high and up to $120 \mathrm{~cm} \mathrm{DBH}$ ), influences the results, since larger phorophytes typically support more-diverse epiphytic floras (Woods et al. 2014) and can accommodate a larger number of rare species.

The vascular epiphytic flora expresses itself in different ecological categories in the relationship established with its phorophytes (Benzing 1990). Brazilian forest epiphyte floras contain a high proportion of characteristic holoepiphytes (83\%) and low proportions of facultative holoepiphytes (7\%), accidental holoepiphytes (5\%) (Kersten \& Silva 2002; Rogalski \& Zanin 2003; Dettke et al. 2008; Buzatto et al. 2008; Kersten \& Kuniyoshi 2009; Mania \& Monteiro 2010; Geraldino et al. 2010; Blum et al. 2011). This composition is due primarily to the marked environmental differences between the canopy and the forest floor, for example the levels of solar radiation, humidity and temperature, which provide different habitats for epiphyte colonization from the base of the trees to the more-exposed branches in the canopy (Nieder \& Zotz 1998). In contrast to forest ecosystems, in rocky environments the contribution of the categories of facultative holoepiphytes (43\%), when evaluating the average of the Menini-Neto et al. (2009), Alves et al. (2008), is similar to that of the characteristic holoepiphytes (44\%) in our study. However, the characteristic holoepiphytes were also the principal component (64\%) on Pseudobombax sp. nov., but the contribution of accidental holoepiphytes (23\%) was more significant than that of facultative holoepiphytes $(10 \%)$. The higher proportion on inselbergs of accidental epiphytic species that can grow both on rocks exposed to full sun and on the trunks and surface roots of trees, sometimes protected by canopies, can be explained by the strong affinity between epiphytic and rupicolous floras in the tropics, especially in South America (Barthlott \& Porembski 2000). The similarity between these floras relates to the xeromorphic conditions on both trees and rocks (e.g., limitation on nutrients and water, high sunlight irradiation, wide swings in temperature and exposure to strong winds), result in similar morphological and physiological adaptations primarily to resist water scarcity, as it directly affects the physiology of the plant (Benzing 1990; Burke 2002).

The enormous climate and geomorphological heterogeneity in the Atlantic Forest Domain, which forms a wide variety of habitats (Rizzini 1979), leads to low similarity between geographically close floras (Menini Neto et al. 2009; Blum et al. 2011). Some investigators have suggested that this low epiphyte similarity is mainly due to the different composition of the orchid family, which is dominant in the canopy of tropical forests (Gentry \& Dodson 1987; Benzing 1990), giving it an important role in similarity indexes of the epiphytic flora (Kersten 2010; Blum et al. 2011).

Our results showed the existence of three distinct floras, which differ especially between 
the areas influenced by montane forests (PP and PR) and the lowest-altitude area, influenced by Semideciduous Submontane Forest (PL) (Fig. 5).

The floristic composition of PP more closely resembles the PR, due to the 30 -shared species (Fig. 6), and the higher richness in PP may be related to its location at an intermediate elevation of the Atlantic Forest Domain (730 m a.s.1.). This relationship to elevation has been observed in several studies of the epiphytic flora in neotropical regions, where the highest richness is associated with intermediate elevations, decreasing toward the highest and lowest elevations (Gentry \& Dodson 1987; Krömer et al. 2007). Another important factor for the higher richness observed in PP may be that the phorophytes are larger (mean dbh $46.4 \mathrm{~cm}$ ) than in the other areas (PL - $35.8 \mathrm{~cm}$ dbh and PR - 22.4 $\mathrm{cm} \mathrm{dbh).} \mathrm{These} \mathrm{differences} \mathrm{agree} \mathrm{with} \mathrm{many} \mathrm{reports}$ that have shown that higher epiphyte richness is associated with large phorophytes, which normally have a longer exposure time, area available for colonization and microhabitat heterogeneity (Hernández-Rosas \& Carlsen 2003; Woods et al. 2014). In particular, for rocky environments, the factors that limit the establishment of plants (e.g., complete or partial absence of soil, low water retention, nutrient shortage) make the species slow-growing and longer-lived (Larson et al. 2000 ), leading phorophytes of these ecosystems support a past flora of the original forests (e.g., vascular epiphytes) that made contact with these environmental elements.

As expected, PL was more dissimilar than PR and PP, since PL lies in a region of drier climate than the other areas and is influenced by the epiphytic flora of the Semideciduous Submontane Forest, which has a lower proportion of epiphytes than the Dense Ombrophilous Forest of the Atlantic Domain (Kersten 2010). As reported by Gentry \& Dodson (1987) that dry-climate regions are generally poor in epiphytic species, whereas ombrophilous areas have the most distinctive epiphyte flora (Benzing 1990). Montane forests (or Cloud forests) are characterized by the frequent incidence of fog and low clouds, and this characteristic promotes greater abundance and species richness of epiphytes in the tropics (Richards 1996).

Only three species were common to all three areas: the bromeliads Aechmea nudicaulis, Tillandsia stricta and the orchid Polystachya concreta. These plants are widely distributed in southeastern Brazil and outside Brazil, occurring in diverse environments (Pabst \& Dungs 1975; Smith
\& Downs 1977, 1979). Similarly, the families that are common to the three inselbergs (Orchidaceae, Bromeliaceae, Polypodiaceae, Cactaceae and Gesneriaceae) are among the epiphyte families with wide distribution associated with the Brazilian Atlantic Rainforest ecosystems (Kersten 2010).

\section{Direct implications for conservation}

The implications of our results for the conservation and management of vascular epiphytes on inselbergs lie in the importance of the architecture and size of the host tree that supports this vegetation. The structure of Pseudobombax sp. nov. promotes the maintenance of epiphytic flora, acting as a refuge for biodiversity on graniticgneissic inselbergs in the Atlantic Domain of southern Espirito Santo state. The high species richness of the vegetation mats and of most other plant communities on eastern Brazilian inselbergs is exceptional when compared to other tropical areas; at last, rocky outcrops usually do not attract much agricultural interest: they have frequently been preserved from human impact and have kept their refugial character (Porembski et al. 1998). However, only one of the three inselbergs is presently within a conservation area, indicating the need for conservation actions for the other areas, as well as adding the endemic species to the lists of endangered flora of Brazil and Espírito Santo. Although do not attract agricultural interest, these results point to the need for stricter oversight of the exploitation of ornamental stones in southern Espírito Santo, where fragile ecosystems and a unique and threatened vascular flora are being destroyed, requiring wthout dash more-detailed study on strategies for maintaining and restoring these ecosystems.

In summary, our data showed that the species richness of vascular epiphytes on Pseudobombax sp. nov. on inselbergs in the Atlantic Forest Domain in southeastern Brazil is higher than that found on quartzite rocky outcrops in the Cerrado Domain and in different forest types of the Atlantic Domain. Exceptions were the higher richness in Dense Ombrophilous Forest and the similar richness of Anthropic Vegetation, both located in southern Brazil. The most diversified ecological category was characteristic holoepiphytes, although with a high proportion of accidental holoepiphytes. The inselbergs located in higher and intermediate elevation areas were more similar to each other and differed from the inselberg at a lower altitude, and members of Orchidaceae and Bromeliaceae 
predominated. The high richness and endemism and the number of endangered species of epiphytes illustrate the important role of Pseudobombax sp. nov., because of its architecture and size, in maintaining biodiversity on the southeastern Brazilian inselbergs. Effective monitoring and management are needed to appropriately conserve the unique and threatened flora of these neglected ecosystems.

\section{Acknowledgements}

The authors express their gratitude to the Coordenação de Aperfeiçoamento de Pessoal de Nível Superior (CAPES), for the Master's scholarship granted to the first author. We are grateful to the many taxonomists, Drs. Alice Calvente Versieux, Elsie Franklin Guimarães, Jefferson Guedes de Carvalho Sobrinho, Marcus Nadruz and Pedro Bond Schwartsburd, for their help in the identification of species. Our thanks to Dr. Vera Lúcia de Moraes Huszar and Andrea Ferreira da Costa (National Museum of Rio de Janeiro), for their important suggestions and review of this manuscript. To Hélio Q.B. Fernandes, curator of the MBML herbarium (Museum of Biology Prof. Mello Leitão), for granting us free access to the collection. To Thaís F. Rodrigues and Waldomiro P. Lopes (ICMBio), for their support for this study in the Caparaó National Park. To Dr. Janet Reid, for the English version. This research was part of the Master's dissertation of the first author from the Postgraduate program in Forest Sciences of the Federal University of Espírito Santo, Brazil. The authors wish to acknowledge the two anonymous reviewers and Marcelo F. Moro, for his detailed and helpful comments and suggestions to the manuscript.

\section{References}

Alves, R.J.V.; Kolbek, J. \& Becker, J. 2008. Vascular epiphyte vegetation in rocky savannas of southeastern Brazil. Nordic Journal of Botany 26: 101-117.

Barthlott, W. \& Porembski, S. 2000. Vascular plants on inselbergs: systematic overview. In: Porembski, S. \& Barthlott, W. (eds.). Inselbergs: biotic diversity of isoleted rock outcrops in tropical and temperate regions. Springer-Verlag, Berlin. Pp. 103-116.

Bataghin, F.A.; Barros, F. \& Pires, J.S.R. 2010. Distribuição da comunidade de epífitos vasculares em sítios sob diferentes graus de pertubação na Floresta Nacional de Ipanema, São Paulo, Brasil. Revista Brasileira de Botânica 33: 501-512.
Bataghin, F.A.; Muller, A.; Pires, J.S.R.; Barros, F.; Fushital, A.T. \& Scariot, E.C. 2012. Riqueza e estratificação vertical de epífitas vasculares na Estação Ecológica de Jataí - área de Cerrado no Sudeste do Brasil. Hoehnea 39: 615-626.

Benzing, D.H. 1990. Vascular epiphytes. Cambridge University Press, New York. 354p.

BFG. 2015. Growing knowledge: an overview of Seed Plant diversity in Brazil. Rodriguésia 66: 1085-1113.

Buzatto, C.R.; Severo, B.M.A. \& Waechter, J.L. 2008. Composição florística e distribuição ecológica de epífitos vasculares na Floresta Nacional de Passo Fundo, Rio Grande do Sul. Iheringia série Botânica 62: 231-239.

Blum, C.T.; Roderjan, C.V. \& Galvão, F. 2011. Floristic composition and altitudinal distribution of vascular epiphytes in the ombrophilous dense forest of the Prata Mountain Range, Morretes, Paraná state, Brazil. Biota Neotropica. Available at <http://www.biotaneotropica.org.br/v11n4/en/ abstract? inventory+bn00811042011>. Access on 14 January 2012.

Brummitt, R.K. \& Powell, C.E. 1992. Authors of plant names. Royal Botanical Gardens, Kew. 275p.

Burke, A. 2002. Island: matrix relationships in Nama Karoo inselberg landscape part II. Are some inselbergs betters sources than others? Plant Ecology 158: 41-48.

Chiodi-Filho, C. 2009. Balanço das exportações e importações brasileiras de rochas ornamentais em 2008. Vol. 3. Abirochas, São Paulo. 23p.

Colwell, R. K. 2013. EstimateS: statistical estimation of species richness and shared species from samples. Version 9. User's Guide and application. Available at $<$ http://purl.oclc.org/estimates $>$. Access on 23 January 2014.

Couto, D.R.; Manhães, V.C.; Favoreto, F.C. \& Faria, A.P.G. 2013. Checklist of the Bromeliaceae from Pedra dos Pontões, Mimoso do Sul, Espírito Santo, Brazil, with four first records for the state. Biota Neotropica 13: 113-120.

Christenhusz, M.J.M.; Zhang, X.-C. \& Schneider, H. 2011. A linear sequence of extant families and genera of lycophytes and ferns. Phytotaxa 19: 7-54.

Dettke, G.A.; Orfrini, A.C. \& Milaneze-Gutierre, M.A. 2008. Composição florística e distribuição de epífitas vasculares em um remanescente alterado de floresta estacional semidecidual no Paraná, Brasil. Rodriguésia 59: 859-872.

Dilcher, D.L.; Lott, T.A.; Wang, X. \& Wang, Q. 2004. A history of tree canopies. In: Lowman, M. \& Rinker, H. (eds.). Forest Canopies. $2^{\text {nd }}$ ed. Elsevier Academic Press, Burlington, New York. Pp. 118-137.

Espírito Santo. 2005. Lista estadual da flora ameaçada de extinção. Decreto de Lei número 1.499-R de 13 de junho de 2005. Diário Oficial, Vitória, Espírito Santo, 14 de junho de 2005. 
Fontoura, T.; Rocca, M.A.; Schilling, A.C. \& Reinert, F. 2009. Epífitas da floresta seca da reserva ecológica estadual de Jacarepiá: Relações com a comunidade arbórea. Rodriguésia 60: 171-185.

Freiberg, M. 1996. Spatial distribution of vascular epiphytes on three emergent canopy trees in French Guiana. Biotropica 28: 345-355.

Gentry A. \& Dodson, C.H. 1987. Diversity and biogeography of neotropical vascular epiphytes. Annals of the Missouri Botanical Garden 74: 205-233.

Geraldino, H.C.L.; Caxambu, M.G. \& Souza, D.C. 2010. Composição florística e estrutura da comunidade de epífitas vasculares em uma área de ecótono em Campo Mourão, PR, Brasil. Acta Botanica Brasilica 24: 469-482.

Giongo, C. \& Waechter, J.L. 2004. Composição florística e estrutura comunitária de epífitos vasculares em uma floresta de galeria na depressão central do Rio Grande do Sul. Revista Brasileira de Botânica 27: 563-572.

Gonçalves, C.N. \& Waechter, J.L. 2003. Aspectos florísticos e ecológicos de epífitos vasculares sobre figueiras isoladas no norte da planície costeira do Rio Grande do Sul. Acta Botanica Brasilica 17: 89-100.

Gotelli, N.J. \& Colwell, R.K. 2011. Estimating species richness. In: Magurran, A.E. \& McGill, B.J. (eds.). Biological diversity: frontiers in measurement and assessment. Oxford University Press, Oxford. Pp. 39-54.

Hammer, Ö.; Harper, D.A.T. \& Ryan, P.D. 2001. PAST: Paleontological statistics software package for education and data analysis. Palaeontologia Electronica 4: 1-9.

Hernández-Rosas, J.I. \& Carlsen, M. 2003. Estructura de las sinusias de plantas del dosel en un portador (Eschweilera parviflora, Lecythidaceae) del bosque húmedo tropical del Alto Orinoco, Estado Amazonas, Venezuela. Ecotrópicos 16: 1-10.

Kersten, R.A. 2010. Epífitos vasculares: Histórico, participação, taxonomia e aspectos relevantes, com ênfase na Mata Atlântica. Hoehnea 37: 9-39.

Kersten, R.A. \& Kuniyoshi, Y.S. 2009. Conservação das florestas na Bacia do Alto Iguaçu, Paraná avaliação da comunidade de epífitas vasculares em diferentes estágios serais. Floresta 39: 51-66.

Kersten, R.A. \& Silva, S.M. 2001. Composição florística e distribuição espacial de epífitos vasculares em floresta de planície litorânea da Ilha do Mel, Paraná, Brasil. Revista Brasileira de Botânica 24: 213-226.

Kersten, R.A. \& Silva, S.M. 2002. Floristica e estrutura do componente epifítico vascular em floresta ombrófila mista aluvial do rio barigui, Paraná, Brasil. Revista Brasileira de Botânica 25: 259-267.

Krömer, T.; Kessler, M.; Gradstein, S.R. \& Acebey, A. 2005. Diversity patterns of vascular epiphytes along an elevational gradient in the Andes. Journal of Biogeography 32: 1799-1809.

Krömer, T.; Kessler, M. \& Gradstein, S.R. 2007. Vertical stratification of vascular epiphytes in submontane and montane forest of the Bolivian Andes: the importance of the understory. Plant Ecology 189: 261-278.

Küper, W.; Kreft, H.; Nieder, J.; Köster, N. \& Barthlott, W. 2004. Large-scale diversity patterns of vascular epiphytes in Neotropical montane rain forests. Journal of Biogeography 31: 1477-1487.

Larson, D.W.; Matthes, U. \& Kelly, P.E. 2000. Cliff Ecology: pattern and process in cliff ecosystems. Cambridge University Press, Cambridge. 340p.

Mania, L.F. \& Monteiro, R. 2010. Florística e ecologia de epífitas vasculares em um fragmento de floresta de restinga, Ubatuba, SP, Brasil. Rodriguésia 61: 705-713.

Martinelli, G. 2007. Mountain biodiversity in Brazil. Revista Brasileira de Botânica 30: 587-597.

Martinelli, G.; Magalhães, C.V.; Gonzalez, M.; Leitman, P.; Piratininga, A.; Costa, A.F. \& Forzza, R.C. 2008. Bromeliaceae da Mata Atlântica brasileira: Lista de espécies, distribuição e conservação. Rodriguésia 59: 209-258.

Menini Neto, L.; Forzza, R.C. \& Zappi, D. 2009. Angiosperm epiphytes as conservation indicators in forest fragments: A case study from southeastern Minas Gerais, Brazil. Biodiversity and Conservation 18: 3785-3807.

Menini Neto, L.; van den Berg, C. \& Forzza, R.C. 2013. Taxonomic revision of Pseudolaelia Porto \& Brade (Laeliinae, Orchidaceae). Acta Botânica Brasilica 27: 418-435.

MMA - Ministério do Meio Ambiente. 2014. Portaria No 443, de 17 de dezembro de 2014 - lista nacional oficial de espécies da flora ameaçadas de extinção. Diário Oficial da União - seção 1, No 245, 18 de dezembro de 2014. Pp. 110-121.

Mori, S.A.; Silva, L.A.M.; Lisboa, G. \& Coradini, L. 1989. Manual de manejo do herbário fanerogâmico. Centro de Pesquisa do Cacau, Ilhéus. 104p.

Nieder, J. \& Zotz, G. 1998. Methods of analyzing the structure and dynamics of vascular epiphyte communities. Ecotropica 4: 33-39.

Obermüller, F.A.; Silveira, M.; Salimon, C.I. \& Daly, D.C. 2012. Epiphytic (including hemiepiphytes) diversity in three timber species in the southwestern Amazon, Brazil. Biodiversity and conservation 21: 565-575.

Pabst, G.F.J. \& Dungs, F. 1975. Orchidaceae Brasilienses Vol. 1. Kurt Schmersow, Hildesheim. 408p.

Pabst, G.F.J. \& Dungs, F. 1977. Orchidaceae Brasilienses Vol. 2. Kurt Schmersow, Hildesheim. 418p.

Perry, D.R. 1978. A method of access into the crowns of emergent and canopy trees. Biotropica 10: 155-157. 
Porembski, S. 2005. Epiphytic orchids on arborescent Velloziaceae and Cyperaceae: Extremes of phorophyte specialisation. Nordic Journal of Botany 23: 505-513.

Porembski, S. 2007. Tropical inselbergs: habitat types, adaptive strategies and diversity patterns. Revista Brasileira de Botânica 30: 579-586.

Porembski, S.; Martinelli, G.; Ohlemuller, R. \& Barthlott, W. 1998. Diversity and ecology of saxicolous vegetation mats on the inselbergs in the Brazilian Atlantic rainforest. Diversity and Distributions 4: 107-119.

Prado, J. et al. 2015. Diversity of ferns and lycophytes in Brazil. Rodriguésia 66: 1-11.

Richards, P.W. 1996. The Tropical Rain Forest. $2^{\text {nd }}$ ed. Cambridge University Press, Cambridge. 600p. Rizzini, C.T. 1979. Tratado de fitogeografia do Brasil. Vol. 2. Hucitec, São Paulo. 374p.

Reis, J.R.M. \& Fontoura, T. (2009). Diversidade de bromélias epífitas na Reserva Particular do Patrimônio Natural Serra do Teimoso - Jussari, BA. Biota Neotropica 9: 073-079.

Rogalski, J.M. \& Zanin, E.M. 2003. Composição florística de epífitos vasculares no estreito de Augusto César, floresta estacional decidual do Rio Uruguai, RS. Revista Brasileira de Botânica 26: 551-556.

Simonelli, M. \& Fraga, C.N. 2007. Espécies da flora ameaçadas de extinção no Estado do Espírito Santo. IPEMA, Vitória. 144p.
Smith, A.R.; Pryer, K.M.; Schuettpelz, E.; Korall, P.; Schneider, H. \& Wolf, P.G. 2006. A classification for extant ferns. Taxon 55: 705-731.

Smith, L.B. \& Downs, R.J. 1977. Bromeliaceae, subfamily Tillandsioideae. Flora neotropica monograph 14. Hafner Press, New York. Pp. 663-1492.

Smith, L.B. \& Downs, R.J. 1979. Bromeliaceae, subfamily Bromelioideae. Flora neotropica monograph 14. Hafner Press, New York. Pp. 1493-2142.

The Angiosperm Phylogeny Group - APG III. 2009. An update of the Angiosperm Phylogeny Group classification for the orders and families of flowering plants: APG III. Botanical Journal of the Linnean Society 61: 105-121.

Veloso, H.P.; Rangel Filho, A.L.R. \& Lima, J.C.A. 1991. Classificação da vegetação brasileira, adaptada a um sistema universal. IBGE, Departamento de Recursos Naturais e Estudos Ambientais, Rio de Janeiro. 124p.

Werneck, M.S. \& Espírito-Santo, M.M. 2002. Species diversity and abundance of vascular epiphytes on Vellozia piresiana in Brasil. Biotropica 34: 51-57.

Woods, C.L.; Cardelús, C.L. \& DeWalt, S.J. 2014. Microhabitat associations of vascular epiphytes in a wet tropical forest canopy. Journal of Ecology. Journal of Ecology 103: 421-430. DOI: $10.1111 / 1365-2745.12357$

Zotz, G. 2013. The systematic distribution of vascular epiphytes: a critical update. Botanical Journal of the Linnean Society 171: 453-481. 
